\title{
Georg Glaeser (Hrsg.): 77-mal Mathematik für zwischendurch - Unterhaltsame Kuriositäten und unorthodoxe Anwendungen
}

Springer 2020, $X+307$ Seiten, ISBN: 978-3-662-61765-6, €22,99; e-Book ISBN: 978-3-662-61766-3, €16,99

\section{Joachim Hilgert}

Angenommen: 14. Januar 2021 / Online publiziert: 28. Januar 2021

(C) Der/die Autor(en) 2021

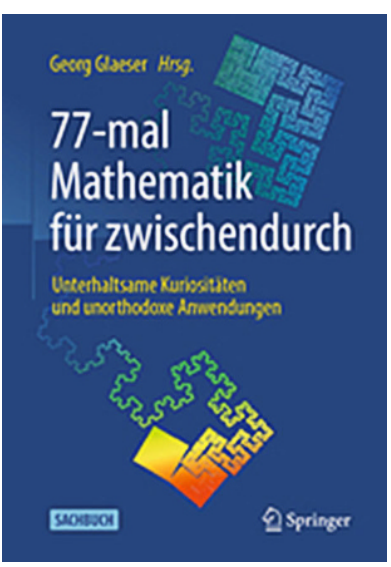

Seit geraumer Zeit gibt es in den verschiedensten Ländern vielfache Aktivitäten, deren Ziel es ist, Interesse an und Verständnis für Mathematik in einem breiteren Kreis der Bevölkerung zu generieren. In Deutschland hat sich die Deutsche Mathematikervereinigung DMV besonders um dieses Anliegen verdient gemacht, und einzelne Autoren wie Ehrhard Behrends haben regelmäßige Kolumnen mit mathematischen Themen geschrieben, die später in dem Buch „Fünf Minuten Mathematik“ in aufbereiteter Form gesammelt wurden.

Das hier zu besprechende Buch ist aus einer österreichischen Initiative hervorgegangen. Einer Idee des 2019 verstorbenen Gilbert Helmberg folgend hat die Österreichische Mathematische Gesellschaft ÖMG seit 2010 rund 100 sogenannter „Mathe-Briefe“ an Interessierte verschickt, in denen unterschiedliche Themen im Umfeld von Mathematik und Mathematikunterricht diskutiert wurden. ,77-mal Mathematik für zwischendurch“ ist eine Auswahl von für diese Veröffentlichung neu editierten Versionen solcher „Mathe-Briefe“. Die einzelnen Beiträge sind thematisch geordnet in den folgenden Rubriken zusammengefasst:

\footnotetext{
J. Hilgert $(\bowtie)$

Universität Paderborn, Paderborn, Deutschland

E-Mail: hilgert@upb.de
} 
I. Algebra und Logik

II. Analysis

III. Geometrie

IV. Zahlentheorie

V. Stochastik

VI. Olympisches

VII.Diverses

Die „Mathe-Briefe“ der ÖMG richten sich ganz explizit an Mathematiklehrerinnen und -Lehrer an höheren Schulen. Das merkt man auch den editierten Texten an, denn fast alle gehen in der einen oder anderen Form auf inhaltliche Bezüge zum Schulunterricht ein, und sei es durch Angabe von an Schüler gerichteter Literatur.

Inhaltlich unterscheidet sich dieser Band nicht grundsätzlich von anderen Sammelbänden zur Popularisierierung von Mathematik. Es gibt Beiträge zu Fibonacci und Adam Ries, zu kubischen Gleichungen, zur Koch-Kurve, zum Newton-Verfahren, zu pythagoreischen Tripeln, zur vollständigen Induktion, zur Spieltheorie und auch einen mit kryptologischem Inhalt. Es gibt aber auch viele Beiträge zu Themen, die ich anderswo noch nicht zur populären Verbreitung aufgearbeitet gesehen habe. Als Beispiel seien G. Glaesers Beiträge zu Stereoskopie und zur Tiefenschärfe von Fotos und L. Summerers Beitrag zu Polynomfunktionen in mehreren Variablen genannt. Auch die biographischen Beiträge haben Überraschendes zu bieten. So war mir die bemerkenswerte Mathematikerin Hilda Geiringer (1893-1973), hier porträtiert von Ch. Binder, vorher nicht bekannt.

Sammelbände zur Popularisierung von Mathematik haben sich in den letzten Jahren zu einem regelrechten Genre entwickelt. Allein im deutschsprachigen Raum sind etliche Bände mit unterschiedlichem Anspruch und unterschiedlichen Schwerpunkten (Anwendungen, Logik und Strukturen, Knobelaufgaben, Wettbewerbsaufgaben, Historisches etc.) erschienen. Ich persönlich habe den Überblick verloren. Während ich bei etlichen Themen des vorliegenden Buches sicher sagen kann, dass sie anderswo auch schon mehrfach behandelt wurden, bleibt mir bei den übrigen Beiträgen nur zu sagen, dass mir nicht bekannt ist, ob sie auch an anderer Stelle zu finden sind. Als Lehrer würde ich mir wünschen, es gäbe eine einschlägige Datenbank, in der ich stöbern könnte, wenn ich Material für eine Unterrichtsreihe oder ein wissenschaftspropädeutisches Projekt suche. Als Forscher haben wir mit dem MathSciNet der American Mathematical Society AMS und dem zbMATH (früher Zentralblatt für Mathematik und ihre Grenzgebiete) solche Datenbanken zur Verfügung.

Ob exklusiv oder nicht, die Beiträge des vorliegenden Bandes sind durchwegs interessant und mit Engagement geschrieben. Manche sind mehr deskriptiv und sehr leicht zu lesen, manche verlangen dem Leser etwas mehr Konzentration ab, und einige setzen zusätzlich zur mathematischen Grundbildung etwas Hintergrund in den Naturwissenschaften, speziell der Physik, voraus. Der aufmerksame Leser findet Kleinigkeiten, die er kritisieren könnte, wie einige stehengebliebene Referenzen aus den urspünglichen „Mathe-Briefen“, die jetzt ins Leere laufen, fehlende Literaturhinweise in einigen Artikeln oder fehlende Erklärungen von für deutsche Leser (die an eine Klassennummerierung von 1 bis 13 gewöhnt sind) irritierende Aussagen wie ,Waren früher solche Probleme bestenfalls in der 6. Klasse beim Kapitel Folgen 
und Reihen und mit Hilfe von Logarithmen lösbar, können heute Schülerinnen und Schüler der 4. Klasse diese Aufgaben bearbeiten." Der Gesamteindruck ist dennoch sehr positiv.

Die durchgehende Berücksichtigung schulischer Aspekte machen das Buch insbesondere auch für engagierte Lehrkräfte interessant. Angesichts der tagtäglichen Herausforderungen, denen sich Mathematiklehrer und -Lehrerinnen in der Praxis konfrontiert sehen, frage ich mich, ob es möglich ist, ihnen noch eine Extrabelohnung anzubieten, wenn sie in einem Akt selbstgesteuerter Weiterbildung einen Aufsatz aufmerksam durchgelesen haben. Vielleicht in Form eines ausgearbeiteten Arbeitsblattes, das sich auf der Webseite des Verlags runterladen kann, wer online drei Fragen zum Text korrekt beantwortet hat.

Funding Open Access funding enabled and organized by Projekt DEAL.

Open Access Dieser Artikel wird unter der Creative Commons Namensnennung 4.0 International Lizenz veröffentlicht, welche die Nutzung, Vervielfältigung, Bearbeitung, Verbreitung und Wiedergabe in jeglichem Medium und Format erlaubt, sofern Sie den/die ursprünglichen Autor(en) und die Quelle ordnungsgemäß nennen, einen Link zur Creative Commons Lizenz beifügen und angeben, ob Änderungen vorgenommen wurden.

Die in diesem Artikel enthaltenen Bilder und sonstiges Drittmaterial unterliegen ebenfalls der genannten Creative Commons Lizenz, sofern sich aus der Abbildungslegende nichts anderes ergibt. Sofern das betreffende Material nicht unter der genannten Creative Commons Lizenz steht und die betreffende Handlung nicht nach gesetzlichen Vorschriften erlaubt ist, ist für die oben aufgeführten Weiterverwendungen des Materials die Einwilligung des jeweiligen Rechteinhabers einzuholen.

Weitere Details zur Lizenz entnehmen Sie bitte der Lizenzinformation auf http://creativecommons.org/ licenses/by/4.0/deed.de. 